\title{
Submaximal angioplasty for symptomatic intracranial atherosclerosis: a prospective Phase I study
}

\author{
Travis M. Dumont, MD, ${ }^{1-3}$ Ashish Sonig, MD, MS, MCh, ${ }^{2,3}$ Maxim Mokin, MD, PhD, ${ }^{2-4}$ \\ Jorge L. Eller, MD, ${ }^{2,3,5}$ Grant C. Sorkin, MD, 2,3 Kenneth V. Snyder, MD, PhD, ,3,3,7 \\ L. Nelson Hopkins, MD, ,3,6,8,9 Elad I. Levy, MD, MBA, ${ }^{2,3,6,8}$ and Adnan H. Siddiqui, MD, PhD $2,3,6,8,9$
}

1Department of Neurosurgery, Division of Surgery, and Department of Medical Imaging, University of Arizona, Tucson, Arizona; Departments of ${ }^{2}$ Neurosurgery, ${ }^{6}$ Radiology, and ${ }^{7}$ Neurology, School of Medicine and Biomedical Sciences, University at Buffalo, State University of New York; ${ }^{3}$ Department of Neurosurgery, Gates Vascular Institute/Kaleida Health, Buffalo; ${ }^{8}$ Toshiba Stroke \& Vascular Research Center, University at Buffalo, State University of New York; and ' $J a c o b s$ Institute, Buffalo, New York; ${ }^{4}$ Departments of Neurology and Neurosurgery, University of South Florida College of Medicine, Tampa, Florida; and ${ }^{5}$ Cerebrovascular Neurosurgery, Oregon Heart and Vascular Institute, Springfield, Oregon

OBJECTIVE Intracranial atherosclerotic disease (ICAD) accounts for approximately 10\% of ischemic strokes. The recent Stenting and Aggressive Medical Management for Preventing Recurrent Stroke in Intracranial Stenosis (SAMMPRIS) study demonstrated a high incidence of perioperative complications (15\%) for treatment of ICAD with stenting. Although the incidence of stroke was lower in the medical arm, recurrent stroke was found in $12 \%$ of patients despite aggressive medical management, suggesting that intervention may remain a viable option for ICAD if perioperative risk is minimized. Angioplasty without stenting represents an alternative and understudied revascularization treatment for ICAD. Submaximal angioplasty limits the risks of thromboembolism, vessel perforation, and reperfusion hemorrhage that were frequently reported with stenting in the SAMMPRIS trial. The authors conducted a prospective Phase I trial designed to assess the safety of submaximal angioplasty in patients with symptomatic ICAD.

METHODS This study was approved by the local institutional review board. Demographic and clinical data were prospectively collected. Angioplasty was performed with a balloon undersized to approximately $50 \%-70 \%$ of the nondiseased vessel diameter in patients with symptomatic ICAD who had angiographically significant stenosis of $\geq 70 \%$. The primary outcome measure was the incidence of periprocedural complications (combined rate of death, stroke, and hemorrhage occurring within 30 days and at 1 year).

RESULTS Among the 65 patients with symptomatic ICAD who were screened, 24 had significant angiographic stenosis that met the inclusion criteria of this study. The mean age was 64.08 years (median 65 years; $S D \pm 11.24$ years), most were men (62.5\%), and most were white (66.67\%). Many patients had concomitants of vascular disease, including hypertension (95.8\%), hyperlipidemia ( $70.83 \%)$, smoking history $(54.1 \%)$, and diabetes mellitus $(50.0 \%)$. Coronary artery disease $(41.66 \%)$ and previous stroke or transient ischemic attack (45.83\%) were frequently present. Most patients (75\%) had anterior circulation stenosis. The mean preprocedure stenosis was $80.16 \%$ (median $80 \%$, range $70 \%-95 \%$ ). Submaximal angioplasty was performed in patients who met the inclusion criteria, with a mean postangioplasty stenosis rate of $54.62 \%$ (median $55.5 \%$, range $31 \%-78 \%$ ). Rates of ischemic stroke in the territory of the treated artery were $0 \%$ within 30 days and $5.55 \%$ (in the only patient who presented with recurrent stroke) at 1 year. The mortality and hemorrhage rates in this series were $0 \%$.

CONCLUSIONS This study demonstrates the safety of the submaximal angioplasty technique, with no permanent periprocedural complications in 24 treated patients.

http://thejns.org/doi/abs/10.3171/2015.8.JNS15791

KEY WORDS intracranial atherosclerosis; angioplasty; stroke; endovascular; vascular disorders

ABBREVIATIONS BI = Barthel Index; COSS = Carotid Occlusion Surgery Study; ICAD = intracranial atherosclerotic disease; $M C A=$ middle cerebral artery; $m R S=$ modified Rankin Scale; NIHSS = National Institutes of Health Stroke Scale; SAMMPRIS = Stenting and Aggressive Medical Management for Preventing Recurrent Stroke in Intracranial Stenosis; $\mathrm{TIA}=$ transient ischemic attack.

SUBMITTED April 7, 2015. ACCEPTED August 12, 2015.

INCLUDE WHEN CITING Published online January 8, 2016; DOI: 10.3171/2015.8.JNS15791. 
A LTHOUGH accounting for a minority of diagnosed ischemic cerebral infarctions, symptomatic intracranial atherosclerotic disease (ICAD) is a dangerous disease, with a relatively high risk for recurrent infarction. ${ }^{8}$ In the Warfarin-Aspirin Symptomatic Intracranial Disease (WASID) trial, the risk of major vascular events was high when patients received either warfarin (8.4 events per 100 patient-years) or aspirin (18.1 per 100 patient-years). ${ }^{3}$ In the Stenting and Aggressive Medical Management for Preventing Recurrent Stroke in Intracranial Stenosis (SAMMPRIS) trial, nearly 1 in 8 symptomatic patients had recurrent stroke within 12 months of observation despite aggressive medical management. ${ }^{2}$ This represented the lowest incidence of recurrent stroke in follow-up of patients with symptomatic ICAD in any prospective study to date. These patients were treated with a combination of aspirin, clopidogrel, rosuvastatin (Crestor; AstraZeneca), and an aggressive lifestyle-changing regimen, including monitored exercise, cessation of smoking, and other healthy lifestyle choices overseen by a lifestyle coach. ${ }^{4}$ Because this treatment may not be a possibility for many patients with symptomatic intracranial atherosclerosis and because the annual stroke rate remains high despite these aggressive interventions, a supplementary treatment strategy of flow augmentation may be considered. Studies to date comparing surgical treatment with bypass (the Carotid Occlusion Surgery Study [COSS ${ }^{11}$ ) or endovascular treatment with stenting (the aforementioned SAMMPRIS $\operatorname{trial}^{2}$ ) have been terminated because of the failure of intervention to demonstrate superior stroke prevention when compared to medical treatment alone.

The failure of intracranial stenting in SAMMPRIS ${ }^{2}$ and cerebral bypass in $\mathrm{COSS}^{11}$ was due primarily to perioperative complications rather than lack of flow restoration. Each study reported a nearly $15 \%$ incidence of perioperative events, including stroke or death. Although it may be difficult to limit complications of surgical bypass with current techniques, the endovascular option of submaximal angioplasty is a viable one compared with stenting to restore flow, while limiting complications. ${ }^{5}$ We conducted a prospective Phase I trial designed to assess the safety of submaximal angioplasty in patients with symptomatic ICAD.

\section{Methods \\ Study Setting and Population}

This study was approved by the University at Buffalo Health Sciences institutional review board. It was designed to have similar inclusion criteria and data end points to those in SAMMPRIS. ${ }^{4}$ Demographic and clinical data were prospectively collected. All patients presenting with stroke were screened for this study consecutively. This study was performed at a single institution, and recruitment took place between January 2012 and October 2014. Data were collected until December 2014.

All patients presenting with stroke or transient ischemic attack (TIA) and in whom ICAD was suspected after review of noninvasive imaging studies (CT angiography and CT perfusion) obtained in the arterial distribution and confirming the patient's stroke syndrome were considered for this study. Patients with a suspected thromboembolic or atheroembolic source located extracranially (including ipsilateral thoracic or cervical vascular occlusive disease) or atrial fibrillation as their ischemic event were not included in this study. Additional exclusion criteria included lacunar stroke; disabling stroke, measured as modified Rankin Scale ( $\mathrm{mRS}$ ) score $>3 ;>30$ days between incidence of presenting stroke/TIA and study enrollment; age $<30$ years or $>80$ years; previous arterial revascularization procedure (including angioplasty, stenting, or endarterectomy of the affected lesion or any area of the vascular tree proximal to the affected lesion); presence of intracranial tumor or vascular malformation; thrombolytic therapy within 24 hours prior to enrollment; progressive neurological signs within 24 hours prior to enrollment; any intracranial hemorrhage within 30 days prior to enrollment; and major surgery within the previous 30 days or planned in the next 90 days after enrollment.

Angioplasty was performed for patients with symptomatic ICAD (stenosis of 70\%-99\% of the diameter of the affected artery) with a balloon undersized to approximately $50 \%-70 \%$ of the nondiseased vessel diameter. For angioplasty, we used the Gateway (Stryker Neurovascular) or the semicompliant cardiac angioplasty balloon (miniTrek, Abbott), with the selection depending on availability. Patients underwent systemic heparinization to obtain an activated coagulation time of $>250$ seconds. The balloon was inflated to its nominal pressure, with an effort to inflate at a rate of $1 \mathrm{~atm}$ per minute and deflate slowly as well, by 1 atm every 30 seconds. With a nominal pressure of $6 \mathrm{~atm}$, this typically accounted for no more than 5-8 minutes of flow cessation per case. Generally, a single balloon inflation angioplasty was performed. All procedures were performed with patients under conscious sedation, using standard transarterial techniques through transfemoral access.

Patients were treated with oral dual antiplatelet therapy prior to the start of the procedure. This included loading doses (if the patient was not already receiving antiplatelet medication) of aspirin (650 mg orally) and clopidogrel (600 mg orally) before the procedure. All patients were tested for response to clopidogrel with the VerifyNow! Assay (Accumetrics). For patients with a poor response to clopidogrel (defined as a response unit of $>220$ ) on the VerifyNow! Assay, clopidogrel dosing was increased from $75 \mathrm{mg}$ once a day to $75 \mathrm{mg}$ twice daily, or an alternate antiplatelet medication was used (prasugrel, $10 \mathrm{mg}$ daily; or ticagrelor, $90 \mathrm{mg}$ twice daily). Dual antiplatelet therapy (typically aspirin, $325 \mathrm{mg}$ daily; and clopidogrel, $75 \mathrm{mg}$ daily) was continued for at least 3 months in all patients, with continued prescription of these medications beyond 3 months at the discretion of the surgeon and stroke neurologist. Patients were additionally treated with rosuvastatin (40 mg daily). Healthy lifestyle choices, including smoking cessation, diet modification, and exercise, were recommended.

\section{Outcome Measures and Baseline Demographics}

The primary outcome measure was the incidence of complications (the combined end point included death, stroke, and hemorrhage) occurring within 30 days and at 1 
year. Stroke was defined as a new neurological deficit that increased the National Institutes of Health Stroke Scale (NIHSS) score by at least 1 point and lasted for more than 24 hours, combined with a corresponding area of infarction on MRI. Neurological examinations were performed by certified research personnel on each patient at regular intervals including at the time of enrollment, periprocedurally, and postprocedurally at 30 days, and at 1 year. The examination included assignment of NIHSS score, mRS score, and Barthel Index (BI). Additional demographic information recorded included age, sex, race, history of typical concomitants of vascular disease (hypertension, diabetes mellitus, hyperlipidemia, cigarette smoking), and relevant medical history (coronary artery disease, previous stroke or TIA).

\section{Statistical Analyses}

Data were described as the mean and median values with range (minimum-maximum) where appropriate. Periprocedural and postprocedural outcomes were presented with respect to the primary combined end point of death, stroke, or hemorrhage within 30 days of the procedure and at 1 year. Procedural data were described as preangioplasty and postangioplasty stenosis. This stenosis was measured as a percentage of narrowing (the minimum measured diameter at the site of greatest stenosis) compared with the normal vessel (the immediately proximal or distal nondiseased segment of the same vessel). Descriptive analysis was performed of categorical (sex, hypertension, diabetes, coronary artery disease, hyperlipidemia, history of stroke) and continuous (age, NIHSS score, $\mathrm{BI}$, and $\mathrm{mRS}$ score) variables. The means were compared by Wilcoxon signed-rank test. Statistical analyses were performed using SPSS V.22 (IBM Statistics).

\section{Results}

Among the 65 patients with symptomatic intracranial atherosclerosis who were screened, 24 had significant stenosis $(>70 \%)$ on angiography that met the inclusion criteria for this study. Demographic data are summarized in Table 1. Among these 24 patients, the mean age was 64.08 years (median 65 years; $\mathrm{SD} \pm 11.24$ years), most were men (62.5\%), and most were white (66.67\%). Many patients had concomitants of vascular disease, including hypertension $(95.8 \%)$, hyperlipidemia $(70.83 \%)$, history of smoking $(54.1 \%)$, and diabetes mellitus (50.0\%). Coronary artery disease $(41.66 \%)$ and previous stroke or TIA (45.83\%) were frequently present. The mean preprocedure performance scores were as follows: NIHSS, 1.08 (median 1, SD \pm 1.34 , range $0-5$ ); BI, 94.79 (median 100, range 65-100); and mRS, 1.08 (median 1, range 0-3) (Table 2).

Details of stenosis percentage and location are provided in Table 2. Most patients (75\%) had anterior circulation stenosis. All patients were treated within 30 days of

TABLE 1. Demographic data in 24 patients with ICAD and angiographic stenosis

\begin{tabular}{|c|c|c|c|c|c|c|c|c|c|}
\hline Case No. & Age (yrs), Sex & Race & HTN & DM & HLD & Smoking & Coronary Artery Disease & Previous Stroke/TIA & Presentation (CVA/TIA) \\
\hline 1 & $77, \mathrm{M}$ & White & Yes & Yes & Yes & Never & Yes & No & TIA \\
\hline 2 & $70, \mathrm{M}$ & White & Yes & No & Yes & Never & Yes & No & TIA \\
\hline 3 & $48, F$ & White & No & No & No & Current & No & No & TIA \\
\hline 4 & $69, \mathrm{M}$ & White & Yes & Yes & No & Never & No & No & CVA \\
\hline 5 & $64, \mathrm{M}$ & Black & Yes & No & Yes & Current & Yes & CVA & CVA \\
\hline 6 & $60, M$ & White & Yes & No & No & Current & Yes & No & TIA \\
\hline 7 & $73, \mathrm{M}$ & White & Yes & No & No & Current & No & No & CVA \\
\hline 8 & $77, \mathrm{~F}$ & White & Yes & Yes & Yes & Never & Yes & CVA & CVA \\
\hline 9 & $48, F$ & White & Yes & No & Yes & Current & No & TIA & TIA \\
\hline 10 & $59, \mathrm{M}$ & Black & Yes & Yes & No & Never & No & TIA & CVA \\
\hline 11 & $44, \mathrm{M}$ & White & Yes & Yes & Yes & Current & No & TIA & TIA \\
\hline 12 & $60, M$ & White & Yes & Yes & Yes & Never & No & CVA & CVA \\
\hline 13 & $66, M$ & White & Yes & No & Yes & Current & Yes & No & TIA \\
\hline 14 & $68, F$ & Black & Yes & No & Yes & Former & No & No & CVA \\
\hline 15 & $76, F$ & Other & Yes & No & Yes & Never & Yes & No & TIA \\
\hline 16 & $80, M$ & Black & Yes & No & Yes & Never & No & No & CVA \\
\hline 17 & $77, \mathrm{~F}$ & White & Yes & Yes & No & Current & No & No & TIA \\
\hline 18 & $74, \mathrm{M}$ & White & Yes & Yes & Yes & Former & No & CVA & CVA \\
\hline 19 & $53, \mathrm{M}$ & White & Yes & No & Yes & Current & No & No & CVA \\
\hline 20 & $55, \mathrm{M}$ & Black & Yes & Yes & Yes & Never & No & No & CVA \\
\hline 21 & $48, F$ & White & Yes & Yes & No & Never & No & TIA & CVA \\
\hline 22 & $54, \mathrm{M}$ & Black & Yes & Yes & Yes & Current & Yes & CVA & CVA \\
\hline 23 & $60, F$ & Black & Yes & Yes & Yes & Current & Yes & CVA & CVA \\
\hline 24 & $78, \mathrm{~F}$ & White & Yes & No & Yes & Never & Yes & CVA & CVA \\
\hline
\end{tabular}

CVA = cerebrovascular accident; $\mathrm{DM}=$ diabetes mellitus; $\mathrm{HLD}$ = hyperlipidemia; $\mathrm{HTN}=$ hypertension . 


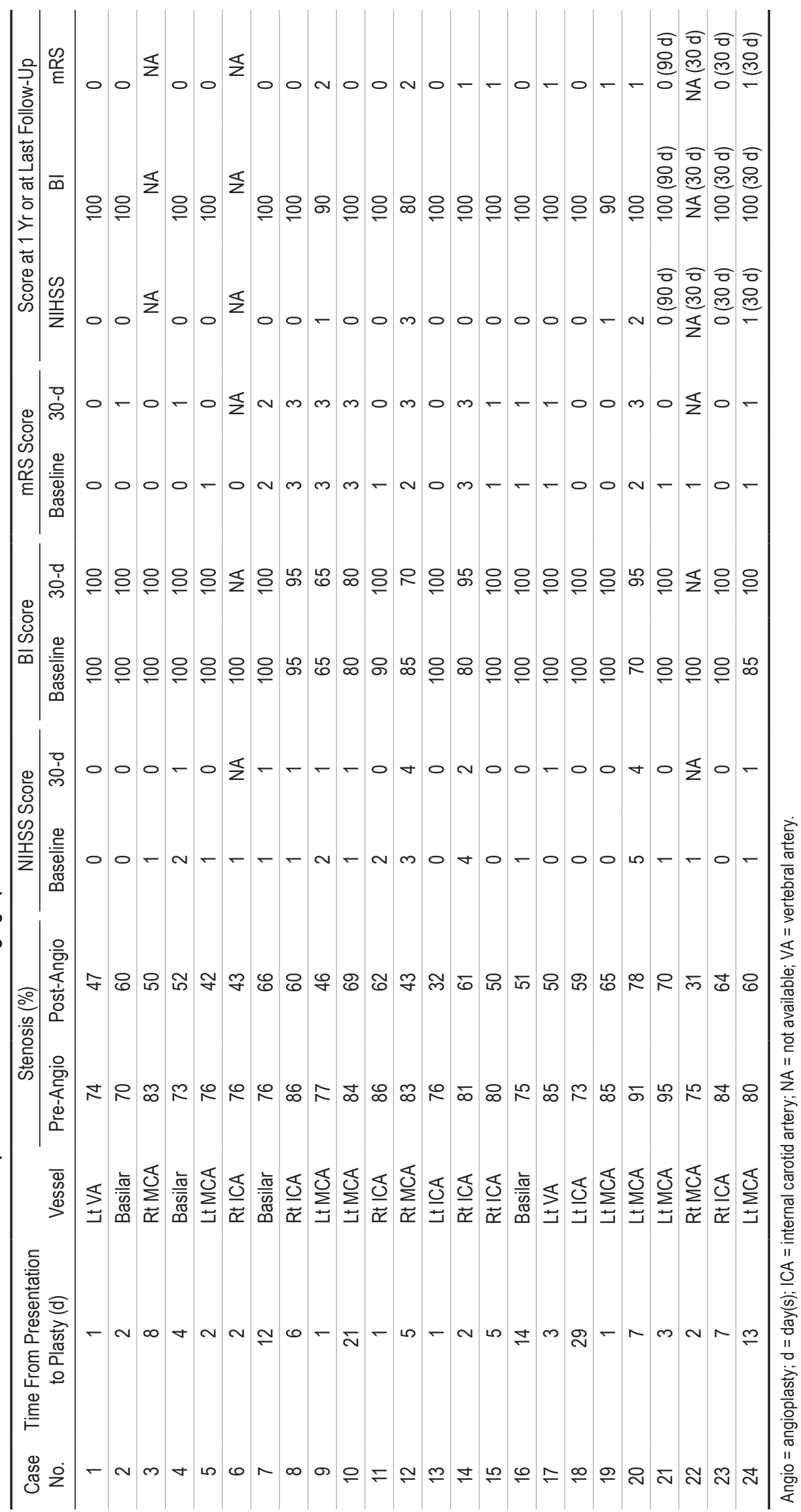


their presenting TIA or stroke, with a median number of days to the procedure of 3.5 (range 1-29 days). The mean preprocedure stenosis was $80.16 \%$ (median $80 \%$, range $70 \%-95 \%$ ), with submaximal angioplasty performed in all patients. Angioplasty was successfully performed in all cases, and the mean postangioplasty stenosis was $54.62 \%$ (median 55.5\%, range 31\%-78\%).

A case-by-case summary of oral antiplatelet and anticoagulant medications before stroke or TIA and after angioplasty is provided in Table 3.

\section{Clinical Outcomes}

Periprocedural Outcome

At the 30-day follow-up period, no patient had an ischemic stroke or death (study end points). Two patients refused 30-day follow-up examination. For the 22 patients available for follow-up examination, the mean 30-day scores were as follows: NIHSS, 0.77 (median 0, range 0-4); BI, 95.45 (median 100, range 65-100); and mRS, 1.18 (median 1, range $0-3$ ). The 30-day rate of stroke and mortality was $0 \%$. Procedural and outcome data are summarized in Table 2. With application of the Wilcoxon signed-rank test, the difference in the means of NIHSS score, BI, and mRS score was not significant when compared with baseline values $(\mathrm{p}=0.106,0.610$, and 0.705 , respectively).

A single perioperative event (a TIA) was noted, with no long-term neurological sequelae in this patient. In this case, the patient had symptoms of ischemia in the distribution of the treated vessel during inflation of the angioplasty balloon (9 minutes), with contralateral hemiparesis and confusion. Symptoms resolved completely within minutes of balloon deflation. No postprocedural residual deficit remained.

\section{Longer-Term Follow-Up}

One-year follow-up was available for 18 of 24 patients and 90-day follow-up for 1 patient (4 of 24 patients were enrolled in the current study within the last 4 months, and 2 of 24 patients were lost to follow-up). At the last followup, the mean scores were as follows: NIHSS, 0.37 (median 0, range 0-3); BI, 97.89 (median 100, range 80-100); and mRS, 0.47 (median 0, range 0-2). At the last follow-up, the difference in means (Wilcoxon signed-rank test) of the NIHSS and mRS scores was significant when compared

TABLE 3. Case-by-case summary of oral antiplatelet and anticoagulant medication*

\begin{tabular}{|c|c|c|}
\hline $\begin{array}{l}\text { Case } \\
\text { No. }\end{array}$ & $\begin{array}{l}\text { Antiplatelet Agents Prior to CVA/ } \\
\text { TIA }\end{array}$ & Post-Angio Antiplatelet Agents \& Duration \\
\hline 1 & None & ASA 325 mg/clopidogrel 75 mg (continued indefinitely) \\
\hline 2 & Warfarin (for PE) & $\begin{array}{l}\text { ASA } 325 \mathrm{mg} / \text { clopidogrel } 75 \mathrm{mg} \text {, warfarin } 3 \text { mos then } \\
\text { ASA } 325 \mathrm{mg} / \text { warfarin (continued indefinitely) }\end{array}$ \\
\hline 3 & ASA $325 \mathrm{mg}$ & ASA 325 mg/clopidogrel 75 mg (lost to follow-up) \\
\hline 4 & None & ASA 325 mg/prasugrel 10 mg (continued indefinitely) \\
\hline 5 & None & ASA 325 mg/clopidogrel 75 mg (continued indefinitely) \\
\hline 6 & None & ASA 325 mg/clopidogrel 75 mg (lost to follow-up) \\
\hline 7 & None & ASA $325 \mathrm{mg} / \mathrm{clopidogrel} 75 \mathrm{mg}$ for $3 \mathrm{mos}$, then ASA alone (continued indefinitely) \\
\hline 8 & ASA $325 \mathrm{mg}$ & ASA $325 \mathrm{mg} /$ clopidogrel $75 \mathrm{mg}$ for $3 \mathrm{mos}$, then ASA alone (continued indefinitely) \\
\hline 9 & ASA $325 \mathrm{mg}$, clopidogrel $75 \mathrm{mg}$ & ASA 325 mg/clopidogrel 75 mg (continued indefinitely) \\
\hline 10 & None & ASA 325 mg/clopidogrel 75 mg (continued indefinitely) \\
\hline 11 & ASA $325 \mathrm{mg}$, clopidogrel $75 \mathrm{mg}$ & ASA 325 mg/clopidogrel 75 mg (continued indefinitely) \\
\hline 12 & None & ASA 325 mg/clopidogrel 75 mg (continued indefinitely) \\
\hline 13 & ASA $81 \mathrm{mg}$ & ASA 325 mg/clopidogrel 75 mg (continued indefinitely) \\
\hline 14 & ASA 325 mg, Plavix 75 mg & ASA 325 mg/clopidogrel 75 mg (continued indefinitely) \\
\hline 15 & ASA 81 mg, Plavix 75 mg & ASA 325 mg/clopidogrel 75 mg (continued indefinitely) \\
\hline 16 & None & ASA 325 mg/clopidogrel 75 mg (continued indefinitely) \\
\hline 17 & None & ASA 325 mg/clopidogrel 75 mg (continued indefinitely) \\
\hline 18 & ASA 325 mg, Plavix 75 mg & ASA 325 mg/clopidogrel 75 mg (continued indefinitely) \\
\hline 19 & None & $\begin{array}{l}\text { ASA } 325 \mathrm{mg} / \mathrm{clopidogrel} 75 \mathrm{mg} \text { until stroke in follow-up, then ASA } 325 \mathrm{mg} / \text { ticagrelor } \\
90 \mathrm{mg} \text { twice daily (continued indefinitely) }\end{array}$ \\
\hline 20 & None & ASA 325 mg/clopidogrel 75 mg (continued indefinitely) \\
\hline 21 & ASA 81 mg, Plavix 75 mg & ASA 325 mg/clopidogrel 75 mg (continued to most recent follow-up) \\
\hline 22 & ASA 81 mg, Plavix 75 mg & ASA 81 mg/clopidogrel 75 mg (continued to most recent follow-up) \\
\hline 23 & Plavix 75 mg & ASA 325 mg/clopidogrel 75 mg (continued to most recent follow-up) \\
\hline 24 & ASA $81 \mathrm{mg}$, rivaroxaban $10 \mathrm{mg}$ & ASA 81 mg/rivaroxaban 10 mg (continued to most recent follow-up) \\
\hline
\end{tabular}

ASA = acetylsalicylic acid (aspirin); $\mathrm{PE}=$ pulmonary embolism.

* All doses were daily unless otherwise noted. 
with baseline values ( $\mathrm{p}=0.012$ and 0.008 , respectively). However, it was nonsignificant for the BI $(\mathrm{p}=0.141)$.

During the postprocedural 30-day follow-up period, 1 patient presented with a TIA. However, no new diffusionweighted imaging changes were seen on MRI and this was not considered a stroke outcome event. None of the patients died during the follow-up period, and only 1 patient suffered an ischemic stroke. This patient had presented with symptomatic stenosis of the left middle cerebral artery (MCA) and underwent submaximal angioplasty. Five months later, he presented with dysarthria and right hemiparesis. A CT angiogram of the head and neck showed left MCA occlusion. The patient underwent an initial submaximal angioplasty followed by placement of a drug-eluting stent (XIENCE Xpedition Everolimus Eluting, Abbott Vascular). Twenty days later the patient presented with worsening of symptoms and in-stent thrombosis, and underwent repeat angioplasty along with the administration of an eptifibatide bolus. The patient's clopidogrel response was found to be nontherapeutic, and he was placed on ticagrelor. At 1-year follow-up, the patient had only mild weakness of the intrinsic muscles of the right hand. His mRS score was 1 and the BI was 90 .

The rate of mortality, hemorrhage, and 30-day stroke was $0 \%$ in this series. Ischemic stroke was seen in only 1 $(5.5 \%)$ of 18 patients during 1 year of follow-up (Fig. 1).

\section{Discussion}

\section{Key Results and Interpretation}

This study demonstrates the safety of submaximal angioplasty in the treatment of symptomatic ICAD. None of the patients suffered ischemic stroke in the 30-day postprocedure period, and there was no hemorrhage or death. The single periprocedural event consisted of a TIA related to prolonged inflation of the angioplasty balloon ( 9 minutes), with return of examination findings to baseline status within minutes after balloon deflation. The rate of

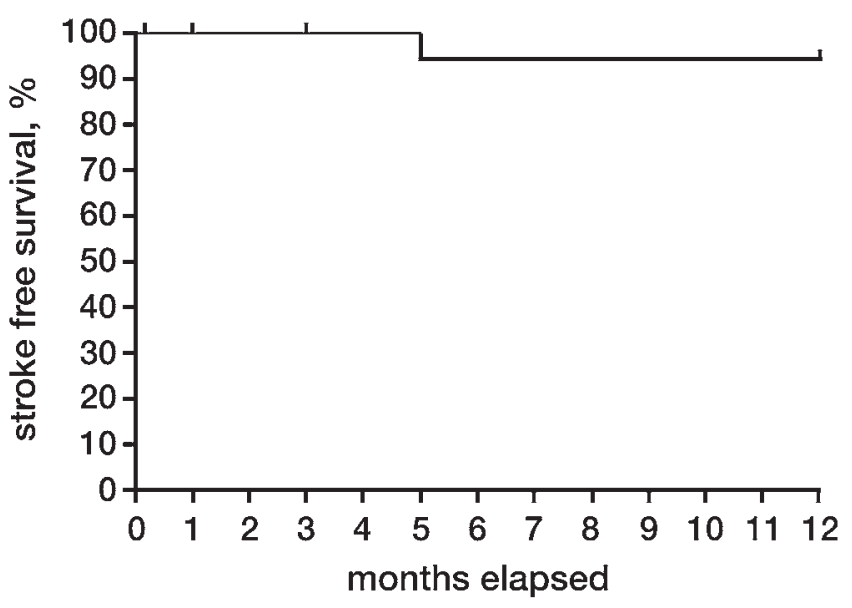

FIG. 1. Stroke-free survival after submaximal angioplasty for symptomatic ICAD. A survival curve demonstrating stroke-free survival among all patients. A single stroke occurred in 1 patient (Case 5) 5 months postprocedure. Patients who were lost to follow-up or with 1-year followup data unavailable are censored with hashmarks. The rate of 1-year stroke-free survival was $94 \%$. periprocedural complications in the present study compares favorably with the nearly $15 \%$ complication profile associated with stenting in a similar patient population in SAMMPRIS, as well as with the 30-day nonfatal stroke risk of $5.3 \%$ in the medical arm of that trial. ${ }^{2}$

Symptomatic ICAD associated with stenosis exceeding $70 \%$ presents because of a number of clinical syndromes, including hypoperfusion in the distal territory, embolic infarct from plaque debris in distal territory, and occlusion of perforator(s) in the region of the plaque. It is the hypoperfusion syndrome that can be aided by flow augmentation, not the other two, which are best treated with antiplatelet and statin therapy. The inclusion of higher-grade stenosis in our study is designed to increase the enrollment of patients with hypoperfusion, who are most likely to benefit from flow augmentation; these patients have been excluded because of the failure of previous attempts to use noninvasive studies (including PET-oxygen extraction fraction in the COSS) ${ }^{11}$ for patient selection. The interventional protocol in SAMMPRIS was modeled on the coronary literature, with an attempt at normalization of underlying vascular anatomy postaugmentation. However, the price of anatomical normalization is an increase in iatrogenic events resulting from local perforator occlusion and distal embolization as well as an increase in procedural complexity with first-generation devices, including stents.

The efficacy of the submaximal angioplasty technique in the setting of ICAD remains unclear. We suspect that patients benefit from this technique due to a marginal but adequate restoration of blood flow. Because flow is proportional to the fourth power of the radius of the affected artery (Poiseuille's equation), a small augmentation in diameter is adequate to restore functional distal territory flow in most cases. Thereafter, we suspect that either medical therapy allows remodeling of underlying plaque or, possibly, the progressive development of pial collateral vessels to distal territory restores normal flow, which may subvert subsequent ischemic infarction due to insufficient flow.

We hypothesize that the low morbidity associated with this procedure is due to the performance of a limited angioplasty. ${ }^{5}$ The submaximal angioplasty technique theoretically allows sufficient flow augmentation through a symptomatic stenotic lesion, with significantly reduced risk of arterial injury, perforator snow-plowing occlusion, and distal embolization; and at the same time it allows a graded return of flow to a more adequate level, which may prevent hyperperfusion syndrome and disastrous intracerebral hemorrhage in the chronically ischemic brain. This in turn may limit the ischemic, technical, and reperfusion complications on the order of $10 \%, 2 \%$, and $2 \%$, respectively, that were seen in SAMMPRIS. ${ }^{2}$ A single patient had an intraprocedural TIA, which we suspect was due to prolonged balloon inflation. To avoid similar events, we recommend balloon inflation for no longer than 5-8 minutes. However, we also believe that slow inflation of $1 \mathrm{~atm}$ per minute and slow deflation of 1 atm per 30 seconds are key to avoiding plaque disruption, with possible dissection and distal embolic events. In our series, in which a slow-inflation submaximal angioplasty technique was used, there were no instances of plaque disruption or dissection with acute vessel occlusion necessitating intraprocedural rescue stenting. It should also be noted that we recommend usage 
of balloons with diameters no greater than $50 \%-70 \%$ of the normal vessel for the same reasons as slow inflation; i.e., to avoid vessel injury and plaque disruption.

The efficacy of the technique in long-term reduction of stroke for patients with symptomatic ICAD who undergo submaximal angioplasty remains unclear. A previous study, the NIH's Wingspan registry, noted a $25 \%$ rate of recurrent stenosis exceeding $50 \%$, with lower rates in the vertebrobasilar circulation and much higher rates in the clinoidal carotid and $\mathrm{M}_{1} \mathrm{MCA}$ segments. Approximately $16 \%$ of these stenoses were responsible for recurrent symptoms. ${ }^{14}$ It is unclear whether this rate was reflective of the stenting strategy and nuances related to vessel apposition or vessel/plaque reaction to the stent implant. In our study, follow-up beyond 30 days was available in 19 cases (1-year follow-up in 18 patients), and only one of them presented with recurrent stroke. This patient was found to have a recurrent stenosis. We attribute this success in stroke prevention in part to concurrent treatment with antiplatelet ${ }^{8}$ and antihyperlipidemic agents. ${ }^{9,12}$ In another US Wingspan registry, ${ }^{1}$ the primary end point (stroke or death within 30 days of the stenting procedure or ipsilateral stroke after 30 days) with high-grade (70\%-99\%) stenosis was $13.9 \% .^{6}$

In the SAMMPRIS trial, the mean follow-up was 11.9 months. ${ }^{2}$ The event rate of ischemic stroke in the territory of the qualifying artery at long term was $10.13 \%$ (23 of 227 patients) in the medical arm. In the stenting cohort, the event rate increased to $16.07 \%$ (36 of 224 patients). In our series, the rate was $5.2 \%$. A similar complication rate was reported by Marks et al. ${ }^{10}$ in their series of 120 consecutively treated patients. Long-term follow-up (mean 42.3 months) was available for 116 patients, and the annual stroke rate was $3.2 \%$. However, their inclusion criteria was $\geq 50 \%$ stenosis, compared with $>70 \%$ stenosis in our study and in the SAMMPRIS trial.

We further hypothesize that the patients with symptomatic ICAD who are most likely to benefit from revascularization treatment with submaximal angioplasty are those who have true flow limitation. A simple measure of vessel stenosis (the inclusion criterion for this study) is a gross simplification of this and does not take into account collateral flow. Although collateral flow may be difficult to measure with the subjective assessment of angiography, objectively decreased flow to the affected area of the brain that is measured by physiological imaging, such as perfusion mapping ${ }^{7,13}$ or quantitative MR angiography, ${ }^{1,15}$ may be of value in determining which patients are at greatest risk for recurrent stroke due to flow limitations from an atherosclerotic lesion. Further prospective study of this technique would benefit from the inclusion of physiological or actuarial measures of diminished flow to identify patients best suited to revascularization, potentially with provocative challenge testing.

\section{Study Limitations}

The principal limitations of this study are its small size and lack of a medical control arm. The true complication rate of submaximal angioplasty may be more appropriately estimated with a larger study. Follow-up angiography was not routinely performed in patients, and thus no measure of recurrent asymptomatic stenosis is known for this patient group. Furthermore, this study was limited to a single high-volume academic center, and similar results may not be encountered universally.

\section{Conclusions}

Submaximal angioplasty for symptomatic intracranial atherosclerotic disease is a safe technique. None of the patients had ischemic stroke in the first 30 days, and only 1 patient presented with recurrent ischemic stroke during 1 year of follow-up. Additional studies with longer follow-up and a larger cohort are required to assess the true safety and efficacy of this technique for reduction of recurrent stroke from ICAD and to determine the patient population that will derive the greatest benefit.

\section{Acknowledgments}

We thank Paul H. Dressel for assistance with preparation of the illustration and Debra J. Zimmer for editorial assistance.

\section{References}

1. Amin-Hanjani S, Du X, Zhao M, Walsh K, Malisch TW, Charbel FT: Use of quantitative magnetic resonance angiography to stratify stroke risk in symptomatic vertebrobasilar disease. Stroke 36:1140-1145, 2005

2. Chimowitz MI, Lynn MJ, Derdeyn CP, Turan TN, Fiorella D, Lane BF, et al: Stenting versus aggressive medical therapy for intracranial arterial stenosis. N Engl J Med 365:993-1003, 2011

3. Chimowitz MI, Lynn MJ, Howlett-Smith H, Stern BJ, Hertzberg VS, Frankel MR, et al: Comparison of warfarin and aspirin for symptomatic intracranial arterial stenosis. N Engl J Med 352:1305-1316, 2005

4. Chimowitz MI, Lynn MJ, Turan TN, Fiorella D, Lane BF, Janis $S$, et al: Design of the stenting and aggressive medical management for preventing recurrent stroke in intracranial stenosis trial. J Stroke Cerebrovasc Dis 20:357-368, 2011

5. Dumont TM, Kan P, Snyder KV, Hopkins LN, Siddiqui AH, Levy EI: Revisiting angioplasty without stenting for symptomatic intracranial atherosclerotic stenosis after the stenting and aggressive medical management for preventing recurrent stroke in intracranial stenosis (SAMMPRIS) study. Neurosurgery 71:1103-1110, 2012

6. Fiorella DJ, Turk AS, Levy EI, Pride GL Jr, Woo HH, Albuquerque FC, et al: U.S. Wingspan Registry: 12-month followup results. Stroke 42:1976-1981, 2011

7. Grandin CB: Assessment of brain perfusion with MRI: methodology and application to acute stroke. Neuroradiology 45:755-766, 2003

8. Holmstedt CA, Turan TN, Chimowitz MI: Atherosclerotic intracranial arterial stenosis: risk factors, diagnosis, and treatment. Lancet Neurol 12:1106-1114, 2013

9. Koskinas KC, Giannoglou GD: Statins, plaque regression, and arterial remodeling: systemic drug effect, local effect, or a combination there of? Am J Cardiol 110:318, 2012 (Letter)

10. Marks MP, Wojak JC, Al-Ali F, Jayaraman M, Marcellus ML, Connors JJ, et al: Angioplasty for symptomatic intracranial stenosis: clinical outcome. Stroke 37:1016-1020, 2006

11. Powers WJ, Clarke WR, Grubb RL Jr, Videen TO, Adams HP Jr, Derdeyn CP: Extracranial-intracranial bypass surgery for stroke prevention in hemodynamic cerebral ischemia: the Carotid Occlusion Surgery Study randomized trial. JAMA 306:1983-1992, 2011 (Erratum in JAMA 306:2672, 2011)

12. Shanmugam N, Román-Rego A, Ong P, Kaski JC: Atherosclerotic plaque regression: fact or fiction? Cardiovasc Drugs Ther 24:311-317, 2010 
13. Wintermark M, Bogousslavsky J: Imaging of acute ischemic brain injury: the return of computed tomography. Curr Opin Neurol 16:59-63, 2003

14. Zaidat OO, Klucznik R, Alexander MJ, Chaloupka J, Lutsep $\mathrm{H}$, Barnwell S, et al: The NIH registry on use of the Wingspan stent for symptomatic 70-99\% intracranial arterial stenosis. Neurology 70:1518-1524, 2008

15. Zhao M, Amin-Hanjani S, Ruland S, Curcio AP, Ostergren L, Charbel FT: Regional cerebral blood flow using quantitative MR angiography. AJNR Am J Neuroradiol 28:1470-1473, 2007

\section{Disclosures}

Hopkins: Grant/research support-Toshiba. Consultant-Abbott, Boston Scientific, Cordis, Covidien. Financial interests-Apama, Augmenix, Axtria, Boston Scientific, Claret Medical Inc., Ellipse, Endomation, Medina Medical, NextPlain, Ostial Corp., Photolitec, Silk Road, StimSox, ValenTx, Valor Medical. Board/ trusteelofficer position-Claret Medical, Inc. Speakers' bureauAbbott Vascular, Toshiba. Honoraria-Complete Conference Management, Cordis, Covidien, Memorial Healthcare System. Levy: Shareholder/ownership interests - Intratech Medical Ltd., Blockade Medical LLC, Medina Medical. Principal investigator-Covidien US SWIFT PRIME Trials. Honoraria for training and lecturing-Covidien. Consultant-Pulsar, Medina Medical, Blockade Medical. Advisory board-NeXtGen Biologics, Stryker. Other financial support-Abbott Vascular for carotid training for physicians. Siddiqui: Research grants (not related to present study) - NIH (co-investigator: NINDS 1R01NS06459201A1 and NIBIB 5 RO1EB002873-07), University at Buffalo (Research Development Award). Financial interests-Hotspur, Intratech Medical, StimSox, Valor Medical, Blockade Medical, Lazarus Effect, Pulsar Vascular, Medina Medical. Consultant-Codman \& Shurtleff, Inc., Covidien Vascular Therapies, Covidien/Medtronic, GuidePoint Global Consulting, Penumbra, Stryker Neurovascular, Pulsar Vascular, MicroVention, Lazarus
Effect, Blockade Medical, Reverse Medical, WL Gore \& Associates. Speakers' bureaus -Codman \& Shurtleff. National Steering Committees - Penumbra 3D Separator Trial, Covidien SWIFT PRIME Trial, MicroVention FRED Trial. Advisory board-Codman \& Shurtleff, Covidien Vascular Therapies, ICAVL, Medina Medical, Inc. Honoraria-Penumbra, Inc., and Toshiba America Medical Systems, for training other neurointerventionists in carotid stenting and for training physicians in endovascular stenting for aneurysms. Snyder: Research, stockholder, and consultant-Boston Scientific. Research, consultant, and financial interest-Cordis, EndoTex, EPI. Research and consultant support-Medtronic, Abbott Vascular, ev3, Toshiba, Zimmer. Research, consultant support, and financial interest-Micrus. Financial interest and stockholder-Access Closure, Inc. Stockholder-Niagara Gorge Medical. Financial interest-Primus. Consultant-Guidant, Kerberos. No disclosures: Dumont, Eller, Sorkin, Sonig, Mokin. No support was received in conjunction with this particular study.

\section{Author Contributions}

Conception and design: Dumont, Sonig, Hopkins. Acquisition of data: all authors. Analysis and interpretation of data: all authors. Drafting the article: Dumont, Sonig. Critically revising the article: all authors. Reviewed submitted version of manuscript: all authors. Statistical analysis: Dumont, Sonig.

\section{Supplemental Information \\ Previous Presentations}

Congress of Neurological Surgeons Annual Meeting, October 19-23, 2013, San Francisco, CA, and Western Neurosurgery Society Annual Meeting, August 16-19, 2014, Sun Valley, ID.

\section{Correspondence}

Adnan H. Siddiqui, Department of Neurosurgery, University at Buffalo, 100 High St., Ste. B4, Buffalo, NY 14203. email: asiddiqui@ubns.com. 